\title{
A versatile wavelength-dependent spectrophotometer for efficiency measurements of CCD and cryogenic gratings
}

Dani Guzman, Sebastien Blais-Ouellette, Marco Bonati, Amal Elgamil

Dani Guzman, Sebastien Blais-Ouellette, Marco Bonati, Amal Elgamil, "A versatile wavelength-dependent spectrophotometer for efficiency measurements of CCD and cryogenic gratings," Proc. SPIE 5499, Optical and Infrared Detectors for Astronomy, (29 September 2004); doi: $10.1117 / 12.551973$

Event: SPIE Astronomical Telescopes + Instrumentation, 2004, Glasgow, United Kingdom 


\title{
A versatile wavelength-dependent spectrophotometer for efficiency measurements of CCD and cryogenic gratings
}

\author{
D. Guzman ${ }^{*}$, S. Blais-Ouellette ${ }^{1}$, M. Bonati ${ }^{1}$, A. Elgamil ${ }^{1}$, \\ ${ }^{1}$ California Institute of Technology, Pasadena, California, USA 91125. \\ *dguzman@astro.caltech.edu; phone 1-626-395-2899, fax 1-626-568-1517.
}

\begin{abstract}
A user-friendly and automatic illuminator with adjustable wavelength and optical power has been developed to obtain precision quantum efficiency $(\mathrm{QE})$ curves of astronomical CCD as well as optical transmission measurements for cryogenic holographic gratings and other optical components. Integrating commercial components with custom mechanical parts and control software, this equipment is able to illuminate a target with light of controlled intensity and wavelength. This facility is primarily intended for testing of Volume Phase Holographic (VPH) gratings at low temperature as well as obtaining CCD quantum efficiencies. A Labview control application runs on a desktop computer allowing full automation of the spectrophotometer. The apparatus includes a Quartz-Tungsten light source, neutral density filters, a monochromator, visible and near-infrared power meters, as well as collimating and focusing optics. Rotation mechanisms allow the characterization of gratings for all angles of diffractions. For CCD testing, network commands allow the facility to get raw images, compute and record QE curves for further detector characterization.
\end{abstract}

Keywords: Spectrophotometer, QE Measurement, VPH testing

\section{INTRODUCTION}

Volume Phase Holographic (VPH) grating and its possible use in astronomical instruments is a technology being studied and characterized in the last years [3,4]. Its high efficiency at room temperature allows thinking of its use at cryogenic instruments [5], for infrared applications. Taking into account the next instruments that the astronomical instrumentation group at the California Institute of Technology will be designing and building [2], a comprehensive testing facility for VPH grating was funded, designed and built.

Taking advantage of the similarity of requirements between this facility and the one required to obtain Quantum Efficiency $(\mathrm{QE})$ curves for imaging detectors like CCDs [6], an optical and mechanical design was conceptualized in order to accomplish both objectives.

The facility is completely automatic and controlled with a LabView application, using advantage of already developed applications for process control and image acquisition [7,13] within the lab. Applications tailored for VPH and QE were programmed. The facility includes a cryogenic chamber to hold a grating at a controlled temperature while is being illuminated, a quartz-tungsten light source, a monochromator and 2 calibrated photodiodes to measure the flux power from the near UV to the NIR, all mounted in motorized rotating stages.

The results of this experiment are presented in [1] for the case of cryogenic VPH gratings.

\section{CRYOGENIC CHAMBER}

The VPH gratings need to be tested at temperatures around $120 \mathrm{~K}$, usual temperature of operation for near infrared instruments [2]. The rest of the setup is at room temperature, so the heat radiation coming from the exterior was a concern for the final temperature of the VPH. A thermal model was implemented to confirm the final temperature of the VPH, standing in front of a window at room temperature. With this model, it was clear that an intermediate cold glass 
next to the VPH was required as radiation shield. One for each side of the VPH conformed what is hereafter called 'filters'. The cryogenic chamber was based on an existing liquid nitrogen dewar. 3D modeling was used to implement a dewar base able to hold the VPH and the filters (through a base plate thermally connected to dewar's liquid nitrogen can), while being optically clear for the experiment. Two windows on the sides holding the vacuum allow this purpose. The 3D model views are presented in figure 1.
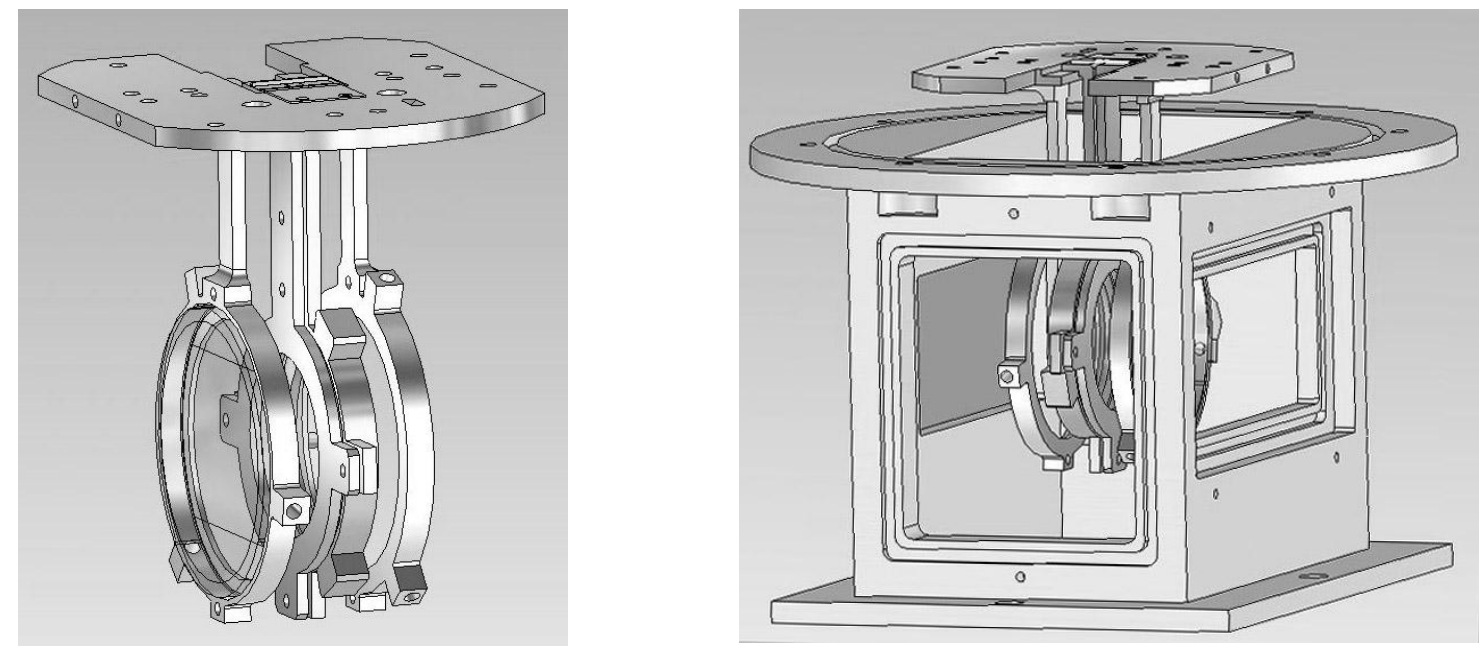

Figure 1: VPH mount, filters and Base plate assembly models: alone and in the cryogenic chamber

The VPH temperature is one of the parameters for the experiment, because it is expected that the VPH efficiency could be modified with temperature, so a temperature control and monitor system was implemented for the cryogenic chamber. A set of temperature sensors and their purpose are described in table 1.

Table 1: Location and Purpose of Temperature Sensors

\begin{tabular}{|l|l|}
\hline Temperature Sensor Location & Purpose \\
\hline Dewar $\mathrm{LN}_{2}$ tube & Monitor empty $\mathrm{LN}_{2}$ condition \\
\hline Base plate & Base temperature (controlled with heater) \\
\hline Filter holder & Monitor temperature gradient \\
\hline VPH holder & Monitor the closest point to the VPH \\
\hline VPH glass (removable) & Monitor the VPH itself (only first VPH) \\
\hline
\end{tabular}

The goal for the temperature control is to keep the VPH at a known and stable temperature. As dozens of VPH will be tested and they are relatively small ( 2 inch diameter), it was impractical to install a temperature sensor on each of them, so as part of the complete thermal characterization of the chamber, the first VPH was installed with a temperature sensor on its center. Figure 2 shows the first thermal test with data from all the sensors, where the VPH reaches about $100 \mathrm{~K}$, considered enough for the experiment, which goal was $120 \mathrm{~K}$.

The base plate has a heater able to deliver $40 \mathrm{~W}$ to the plate. It was tested that $40 \mathrm{~W}$ were able to increase the temperature in $60 \mathrm{~K}$, enough for the scope of the experiment. Go to [1] for details about chosen temperatures. 


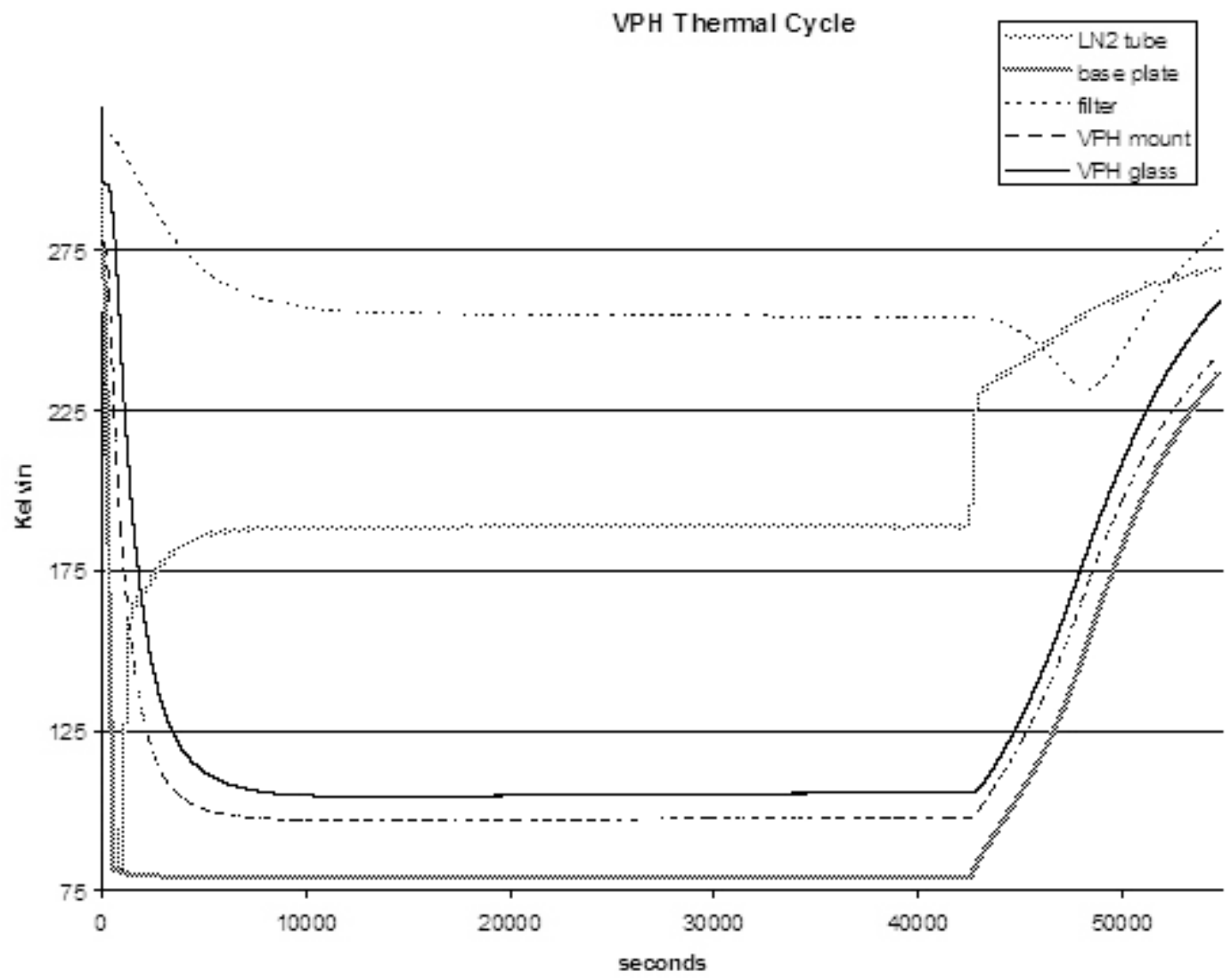

Figure 2: Experimental thermal cycle of the cryogenic chamber

\section{OPTICAL COMPONENTS}

The facility uses commercial components assembled on an optical bench. The components are common to spectrophotometric measurements and several references $[5,6,8,9,10]$ describe similar setups. A list of major parts with description can be found in table 2 .

Table 2: Optical Components

\begin{tabular}{|l|l|}
\hline Component & Description \\
\hline Light source Oriel model 66892 & $\begin{array}{l}\text { Light head w/quartz tungsten lamp, housing and } \\
\text { power supply }\end{array}$ \\
\hline Filter wheel Oriel model 74041 & Neutral Density (ND) filters \\
\hline Monochromator Oriel model 74100 & Wavelength selection \\
\hline $\begin{array}{l}\text { Calibrated \& cooled Si detector Oriel } \\
\text { model 71642 }\end{array}$ & Visible measurement \\
\hline $\begin{array}{l}\text { Calibrated \& cooled InGaAs detector } \\
\text { Oriel model 71646 }\end{array}$ & NIR measurement \\
\hline Optical Power Meter Oriel model 70310 & Detector reading \\
\hline
\end{tabular}

Figure 3 presents a general view of the optical bench and the optical components of the facility. 


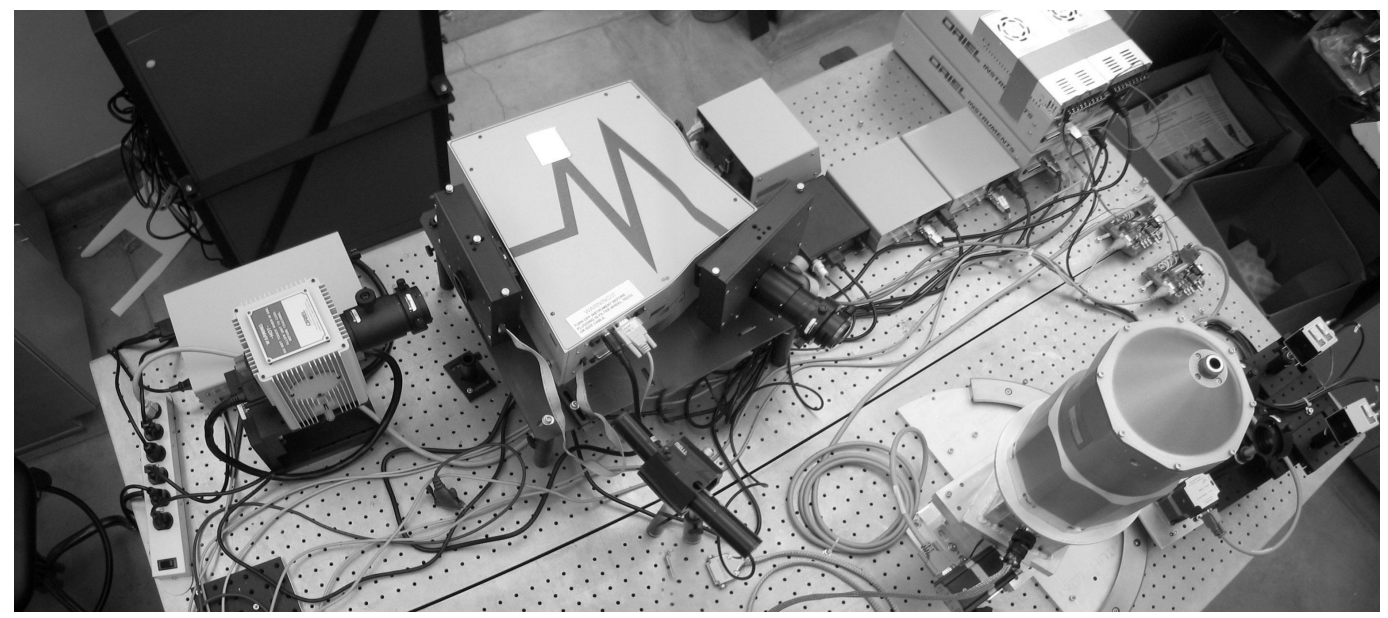

Figure 3: Aerial view of the facility, showing light source, monochromator and cryogenic chamber

Some remarks of this particular setup are:

- The light source includes optical feedback stabilization to minimize recalibration along one run because of variation in intensity of the source.

- The beam from the monochromator is collimated.

- There are two detector elements (photodiodes) to cover the visible and near infrared regions of the spectrum. Each of them is independently read.

- Each detection sensor has a lens to focus the beam on its sensible area.

- The monochromator-chamber-sensors set is covered by a light-tight enclosure, to avoid scattered light from the light source or other sources in the lab.

\section{VPH CHARACTERIZATION REQUIREMENTS}

To completely characterize a transmitting grating like a VPH, the element needs to be illuminated by a monochromatic beam at a selectable angle respect to the element. The beam will be diffracted as a function of the grating properties and the wavelength chosen [11]. This physical behavior implies that, for a complete characterization at different angles, the VPH must rotate along its axis as well as the detection sensors, i.e. having two degrees of freedom. This was accomplished using a rotating stage underneath the cryogenic chamber and a carriage sliding on a curved track, carrying the detection sensors, hereafter called detector carriage. 


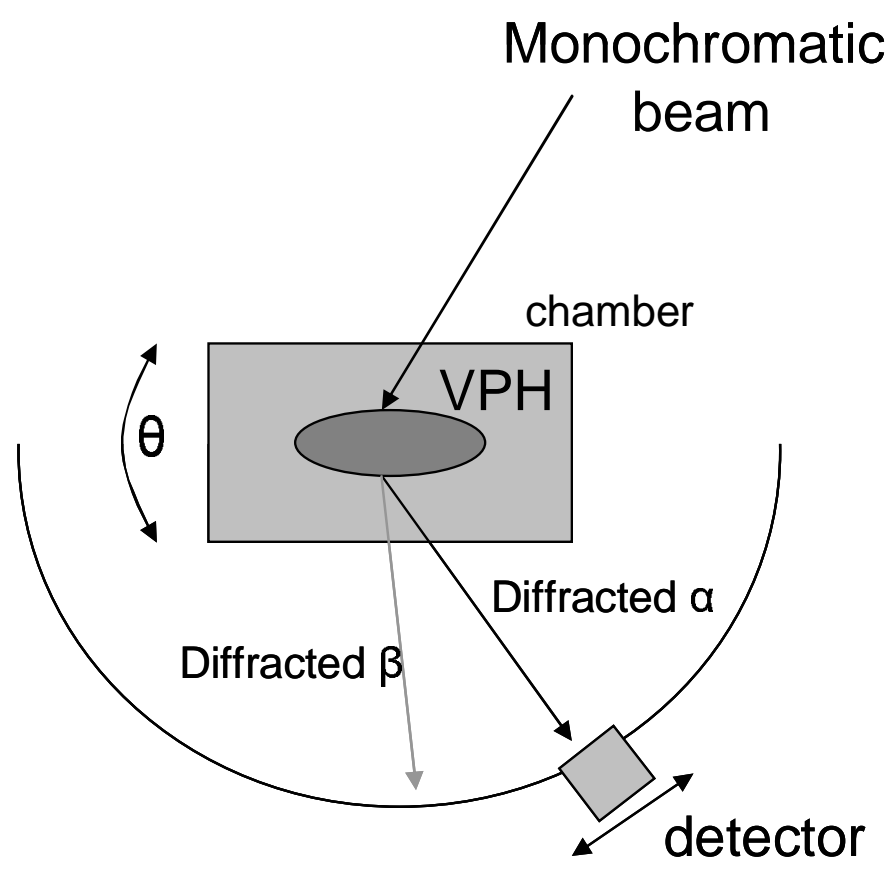

Figure 4: Plant view of the VPH rotational scheme

The rotational concept is schematized in figure 4. For a fixed angle $\theta$, the monochromatic beam at a wavelength $\lambda_{\alpha}$ illuminates the VPH, being diffracted at a certain angle $\alpha$. For another wavelength $\lambda_{\beta}$, the diffracted angle will be $\beta$. To measure both diffracted beams, the detector needs to rotate, centered respect to the VPH axis. $\theta$ and $\alpha$ (or $\beta$ ) are the two degrees of spatial freedom of the system.

Smart motors from Quick Silver Controls were used to control both degrees of freedom. Considering that the VPH ruling could not be perfectly vertical respect to the bench, one could have a misalignment in height of the beam for different detector carriage angles, thus a low profile manual tip-tilt table was added underneath the chamber, to allow further adjustment of the height of the beam respect to the bench, in order to have the detection sensors aligned to the beam for all angles.

The procedure to get the efficiency for a VPH is described in [1].

\section{QE MEASUREMENT MODE}

The facility can be reconfigured to measure $\mathrm{QE}$ for imaging detectors, like CCDs. The goal for this purpose is to illuminate the imaging detector and the detection sensors with the same beam. This is accomplished replacing the cryogenic chamber by a flat mirror, to point the collimated beam towards the photodiodes detectors or to the imaging detector under test. As the lenses cannot be used in a direct manner, the collimated beam is flattened with an integrating sphere added at the output of the monochromator. The detector under test is placed at the same distance than the detection sensors. For this application the monochromator's built-in shutter is used to expose the imaging detector to the monochromatic light.

The expression to obtain the $\mathrm{QE}$ of an imaging detector is based on the formula given in [12], modified for the case of a calibrated detector, which delivers a power reading. 


$$
Q E_{C C D}=\frac{S_{C C D}}{P_{S}} \cdot \frac{A_{D} \cdot 12390 \cdot q}{\lambda \cdot W_{D}}
$$

Where $S_{C C D}$ is the mean signal in the area of analysis of the image from the $\operatorname{CCD}\left(e^{-}\right), P_{S}$ is the individual pixel area

$\left(\mathrm{cm}^{2}\right), A_{D}$ is the photodiode sensor area $\left(\mathrm{cm}^{2}\right), q$ is the electron charge, $\lambda$ is the wavelength selected $(\AA)$ and $W_{D}$ is the power reading (Watt).

One of the important features of any automatic QE measurement facility is the ability to measure the level of the signal in the image prior taking the final image for the QE sequence, in order to keep it within a predefined range and avoid saturation. Because of the no-flat spectral response of the light source and the imaging detector, for different wavelengths the amount of electrons collected will vary enormously, for a fixed exposure time. It is a goal for a $\mathrm{QE}$ measurement facility to adjust the exposure time and/or modify the intensity of the light source, through the Neutral Density filter wheel, to avoid any saturation in the image. An iterative process is performed in this facility to obtain every image of a $\mathrm{QE}$ sequence within a user-defined range.

\section{CONTROL SOFTWARE}

The software architecture was designed to reach maximum modularity and flexibility. It consists of several independent software modules, where each module handles a specific piece of the system. The software developed for the QE testing facility is explained here, while the one developed for VPH testing is explained in [1].

Labview was chosen as the programming language because of its flexibility as well as availability of most of the lowlevel drivers for the different hardware subsystems. Furthermore, many of the image acquisition systems in the lab are based in this platform as well.

The architecture is based on a central server, which listens for ASCII commands from an external client through a raw $\mathrm{TCP} / \mathrm{IP}$ socket. The client for this application is a main GUI or any scripting language. The server re-sends the command to the appropriate module, to execute the action and return a response. This central server is an ArcVIEW server [13], to take advantage of all the variable management already developed in this application. Useful information like image headers, log files and recreation of test conditions are stored in an internal database, also accessible externally through a network port.

The server port is used by the external client to request commands and/or information. There will be normally required actions (move filter wheel, move mirror, etc) as well as information (for instance, data for the image headers) which can be requested to the database using a direct network connection.

Besides the normal TCP/IP port of the server and the server database, many of the subsystem modules have their own external ports; therefore they can be used or tested in a completely independent way.

The subsystems described below use RS-232 serial ports.

Quicksilver module: talks to a Quicksilver smart motor for mirror motion. The basic Labview drivers were done at CTIO and completed at Caltech.

Monochromator module: talks to the Oriel monochromator. It uses Oriel drivers.

ND Filter Module: moves the Oriel ND filter wheel. The Labview drivers were done at Caltech based on the ASCII commands provided by Oriel.

Detectors module: reads and sets the calibrated Oriel Detectors, through the Optical Power Meter. The Labview drivers were done at Caltech based on the ASCII commands provided by Oriel. 


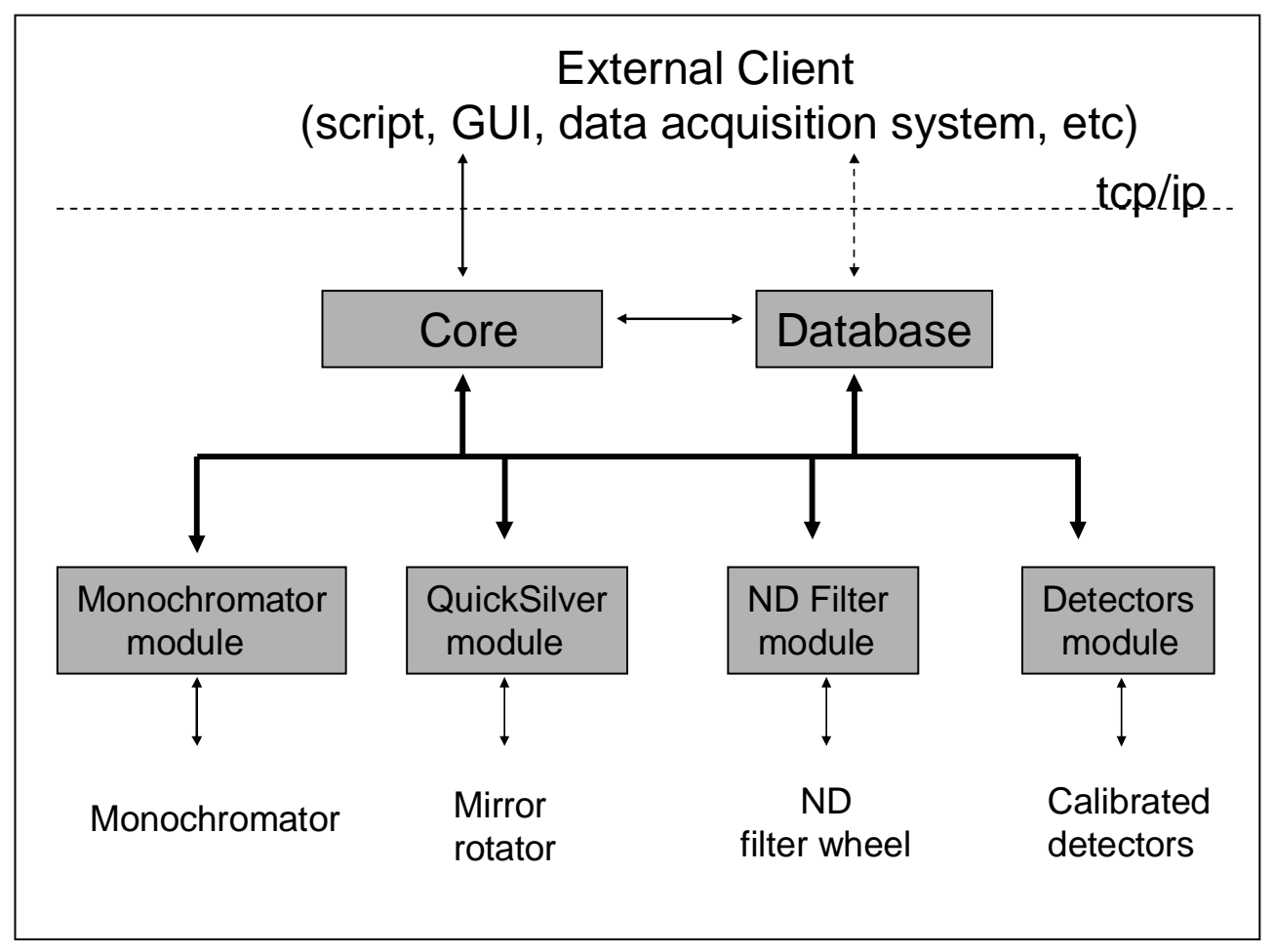

Figure 5: Software Architecture

As it can be seen in figure 5, the "intelligence" for coordinating the different actions is not currently embedded in the instrument software; it is in the client side where the "sequence of actions" lives. This external client can be a script, a coordinating GUI or an external CCD data acquisition system. An optional "coordinating module" can also be added at the server side if needed, but leaving it external gives flexibility for using it with different data acquisition systems.

A fully automated QE testing procedure can be done by using an external script (or GUI) which:

- Leaves in place all the parts needed in order to get a detector measurement under well-known conditions (wavelength, filter, etc) by talking to the "instrument software".

- $\quad$ After moving the mirror to the CCD position, asks the CCD camera software to take an image.

- If the script is written in some language with embedded image analysis tools (like IRAF, IDL, MATLAB, etc), then the script can follow the automation, presenting a QE plot as a result.

The implementation of this architecture for this facility was based in IRAF scripting.

\section{CONCLUSIONS}

A versatile facility for optical efficiency of VPH gratings and quantum efficiency of CCD was presented. Further usage of this setup is easy to ambition, because the rotating concept and monochromatic illumination has multiple applications in optical and chemical characterization. 


\section{REFERENCES}

1. S. Blais-Ouellette, D. Guzman, A. Elgamil, "Cryogenic VPH Gratings for CELT/TMT", This Proceedings, Glasgow, 2004.

2. “Keck Infrared Multi Object Spectrograph. Conceptual Design Study Documentation”, K. Taylor, Principal Investigator, California Institute of Technology, March 2002.

3. S. Barden, J. Arns, W. Colburn, "Volume-phase holographic gratings and their potential for astronomical applications", Optical Astronomical Instrumentation, Proc. SPIE Vol. 3355, p. 866-876. 1998

4. S. Barden, J. Arns, W. Colburn, J. Williams, "Evaluation of volume-phase holographic grating technology", Optical Spectroscopic and Instrumentation for Atmospheric and Space Research IV, Allen M. Larar, Martin G. Mlynczak, Proc. SPIE Vol. 4485 p. 429-438, Jan 2002.

5. GOLem-OABr, "Preliminary test on VPH in cryogenic environment", Web Reference: http://golem.merate.mi.astro.it/projects/vph/cryo/cryo.html .

6. M. P. Lesser, B. L. McCarthy, "Quantum Efficiency Characterization of Scientific CCDs", Solid State Sensor Arrays and CCD Cameras, Constantine N. Anagnostopoulos, Morley M. Blouke, Michael P. Lesser, Proc. SPIE Vol. 2654, p. 278-286, Mar 1996.

7. M. Bonati, M Ashe, "ISPI Software. An Infrared Application of ArcVIEW System", Scientific Detectors for Astronomy, Paola Amico, James W. Beletic, Jenna E. Beletic, p. 427-430, Kluwer Acedemic Publishers, Waimea, 2002.

8. N. Tamura, G. J. Murray, P. Luke, C. Blackburn, D. J. Robertson, N. A. Dipper, R. M. Sharples, J. R. AllingtonSmith, "Cryogenic Tests of Volume-Phase Holographic Gratings: I. Results at 200 K", Exper. Astron, Nov 2003.

9. Dahong D. Qian, Anthony DeSimone, Stuart Boyd, Richard Young, William E. Schneider, "A Comprehensive Illuminator for Solid-State Image Sensor Testing and Characterization", Current Developments in Optical Design and Engineering VII, Proc. SPIE Vol. 3429, p. 112-116, Oct 1998.

10. G. Bonanno, P. Bruno, A. Cali, R. Cosentino, R. Di Benedetto, M. Puleo, S. Scuderi, "The Catania Astrophysical Observatory facility for UC CCD characterization" EUV, X-Ray, and Gamma-Ray Instrumentation for Astronomy VII, Oswald H. Siegmund, Mark A. Gummin Proc. SPIE Vol. 2808, p. 242-249, Oct 1996.

11. J. A. Arns, W. S. Colburn and S. C. Barden, "Volume phase gratings for spectroscopy, ultrafast laser compressors, and wavelength division multiplexing", Current Developments in Optical Design and Optical Engineering VIII, Robert E. Fischer, Warren J. Smith, Proc. SPIE Vol. 3779, p. 313-323, Oct 1999.

12. J. Janesick, Scientific Charged Couple Devices, p. 152-153, SPIE Press, 2001.

13. M. Ashe, M. Bonati, S. Heathcote, "ArcVIEW: A LabVIEW-based astronomical instrument control system", Advanced Telescope and Instrumentation Control Software II, Hilton Lewis, Proc. SPIE Vol. 4848, p. 508-518, Dec. 2002. 